\title{
Determinants of spatial priming in environmental memory
}

\author{
RICHARD C. SHERMAN and KAM M. LIM \\ Miami University, Oxford, Ohio
}

\begin{abstract}
Spatial priming in recognizing objects in experimentally learned environments has been proposed as strong evidence for spatial organization of environmental memory. However, in all studies showing recognition priming effects, encoding and rehearsal contiguity may have coincided with spatial proximity, and thus priming may have been due to temporal associations formed during rehearsal, not encoded spatial relations per se. We investigated this question in four experiments, using a trip trial learning method in which temporal contiguity and spatial relations were independent. In Experiment 1, no spatial priming in recognition was found, even though indirect evidence suggested that subjects had encoded spatial relations. In Experiment 2, the trip trial method was compared with the free study procedure commonly used in previous priming studies. Spatial priming occurred only for free study subjects, even though the two groups were equivalent on direct measures of encoding accuracy. In Experiment 3, spatial priming in recognition was obtained with a modification of the trip trial method in which temporal and spatial contiguity were deliberately confounded. In Experiment 4, the unmodified trip trial method produced spa. tial priming in a location-decision task. Taken together, our results suggest that environmental memory may be spatially organized, but retrieval of object identities does not necessarily activate encoded spatial relations.
\end{abstract}

Storing, retrieving, and manipulating information about the environment are essential for the successful completion of many of our daily tasks. Wayfinding and navigation, for example, require memory for spatial and structural information, such as distance and bearing relations, routes between locations, demarcations of regional boundaries, and the distinguishing physical features of landmarks and destinations. Understanding how these various types of information are represented and organized in memory is an important goal.

Considerable early research on factors influencing the accuracy of encoded spatial information in environmental memory showed that distance and bearing relations among objects are generally well preserved (e.g., Evans, 1980; Merrill \& Baird, 1987). However, as several investigators have recently pointed out (Clayton \& Chattin, 1989; McNamara, 1986; Merrill \& Baird, 1987), the fact that distance and bearing information is represented in memory does not necessarily mean that environmental memory is spatially organized, and current research has tended to focus on questions of structure, as distinst from questions of content.

An important finding in this regard is the spatial priming effect observed in certain memory tasks involving environmental information. For example, the recognition of an object as a member of an environmental array is facili-

Portions of this research were presented to the American Psychological Society Convention held in Dallas on June 10, 1990. Requests for reprints should be sent to Richard C. Sherman, Department of Psychology, Miami University, Oxford, OH 45056. tated if the immediately preceding object is close to the current target in terms of Euclidean or functional distance (McNamara, 1986; McNamara, Altarriba, Bendele, Johnson, \& Clayton, 1989; McNamara, Ratcliff, \& McKoon, 1984), or if it is from the same subregion of the environment (McNamara, 1986; McNamara, Hardy, \& Hirtle, 1989; Sherman, 1987). Since a recognition task does not explicitly require retrieval or manipulation of spatial information, these priming effects seem to offer strong evidence that the representation of encoded identities of objects in the environment is spatially organized. Further, the regional priming effects in these studies have been interpreted as supporting a particular class of memory models in which environmental information is organized hierarchically - for example, as a graph-theoretic tree in which individual locations are linked to regional nodes by "containment" relations (see McNamara, Hardy, \& Hirtle, 1989; Stevens \& Coupe, 1978).

However, there is reason to question whether the priming effects observed in these investigations are in fact due solely to encoded spatial relations. In all previous studies that have yielded recognition priming effects, researchers have used experimentally learned configurations (i.e., novel maps or layouts of objects in an experimental space), in which encoding and rehearsal contiguity may have coincided with spatial proximity. ${ }^{1} \mathrm{~A}$ typical procedure (see, e.g., McNamara, 1986; McNamara, Altarriba, et al., 1989) has been to allow subjects to study the experimental configuration for a brief period and then test their knowledge of object locations, repeating the study/test sequence until some accuracy criterion is reached. Although 
the behavior of subjects during the study/test period has not been described, a likely strategy would be to encode and rehearse proximal groupings of objects together, thus producing strong associative links among them that would be coincidental with spatial proximity and common regional membership (Clayton \& Chattin, 1989; Clayton \& Habibi, 1991). Thus, the recognition priming effects previously observed in studies with experimentally learned configurations may be due to associations arising from temporal contiguity during rehearsal, not to encoded spatial relations per se. If this is correct, the inferences drawn from these studies regarding the organization and structure of environmental memory must be reexamined.

Indirect evidence for our interpretation comes from several recent studies in which it has been shown that spatial priming does not occur in naturally learned environments (e.g., college campuses or city layouts) unless the task requires a spatial decision (Clayton \& Chattin, 1989; McNamara, Altarriba, et al., 1989), or unless spatial proximity coincides with functional relatedness (Merrill \& Baird, 1987). We suggest that this is because learning everyday environments typically involves varied movement patterns and multiple start/destination pairings of locations that reduce the coincidence between temporal and spatial proximity. In this case, simple recognition of an object as a member of a particular configuration can be made on the basis of nonspatial qualities, such as the general familiarity with object identities or their functional relatedness, without reference to spatial relations per se. A locational task, however, would more likely activate spatial associations between objects.

Our analysis differs in certain key respects from other interpretations that have been proposed. In particular, McNamara, Altarriba, et al. (1989) have suggested that the reason recognition leads to spatial priming in experimental but not natural situations is that when environments are learned in an experimental setting, the identities and locations of objects are experienced only in the same spatial context. Decisions about an object's being a member of the environmental array "must be based on these highly contextualized and necessarily spatial experiences (p. 447)." On the other hand, when environments are learned naturally, identities and locations are experienced separately in many different contexts, some of them nonspatial, and therefore recognition decisions can be based on sources of familiarity that are not spatial.

According to this "restricted context" view, how an experimental configuration is learned should make little difference in recognition priming, provided that identities and locations are experienced in a single, spatial context. However, if our analysis is correct, having subjects acquire knowledge of an experimental configuration by means of a learning procedure that reduces the coincidence between spatial relations and rehearsal contiguity should produce spatial priming effects in tasks that involve location decisions, but not simple recognition. We examined this possibility in the four experiments reported below.
In Experiment 1, spatial priming in a recognition task was examined with subjects who learned an experimental layout in a series of trip trials in which the paths and start/destination pairs were randomly varied on each trial, thus reducing the association between exposure sequence and spatial proximity. The learning procedure and materials were similar to those in an earlier study (Sherman, 1987 ) in which we observed recognition priming effects with objects in the same region of an environmental configuration. However, in that study, subjects were exposed to objects one region at a time, thereby possibly confounding regional and temporal rehearsal associations in the same manner as in the study/test method described above. In Experiment 1, we eliminated this confound. In Experiment 2 , we compared recognition priming in two groups of subjects who differed in how they learned the same experimental layout. One group learned the configuration in a manner similar to that in the study/test method used in previous studies (e.g., McNamara, Altarriba, et al., 1989). The other learned the layout by the same trip trial method that was used in Experiment 1. In Experiment 3, we examined the spatial priming effects produced by a modification of the trip trial method that deliberately confounded spatial and temporal contiguity. In Experiment 4, we investigated spatial priming in a location-decision task with subjects who learned the configuration with the original trip trial method. In all four experiments, we obtained a converging measure of memory organization by examining subjects' free recall protocols of object names, and in Experiments 2-4, we assessed subjects' accuracy of encoded spatial relations using distance estimations and sketch maps.

\section{EXPERIMENT 1}

\section{Method}

Subjects. Sixteen undergraduates, 8 men and 8 women, participated in return for research credit in introductory psychology courses.

Stimulus environment. The experimental layout in these studies is similar to that used in a number of our earlier investigations of environmental memory (Sherman, 1987; Sherman, Croxton, \& Smith, 1979; Sherman, Oliver, \& Titus, 1980). In designing the stimulus configuration, we considered two factors that have often been ignored in research done with experimental layouts. First, we attempted to simulate situations that require movement and physical interaction with the environment to obtain location information, as distinct from learning maps or map-like arrays from a single fixed orientation. Second, the structure of our environment was based on the idea that many everyday environments are divided into subareas that are sometimes subsumed by larger regions, and objects in different regions are often not visible from one another, either because of distance or because of the nature of region boundaries (e.g., walls dividing the interior of a building).

A diagram of the experimental environment is shown in Figure 1. It consisted of a 6.4-m-square room divided into two equal major regions ( $A$ and $B$ on the diagram) by an opaque orange screen $1.8 \mathrm{~m}$ high and $3.6 \mathrm{~m}$ long. The designations " $\mathrm{A}$ " and " $\mathrm{B}$ " are for descriptive purposes only - they were not labels learned by the subjects. Region A was divided into two smaller subareas (A1 and A2) by a $1.8 \times 1.8 \mathrm{~m}$ opaque black divider placed at a right angle to 


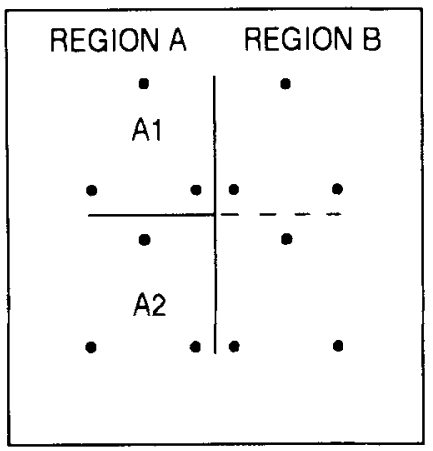

Figure 1. A diagram of the 6.4-m-square stimulus environment. Solid dots represent stimulus locations. Solid lines represent physical boundaries. The dotted line in region B is for descriptive purposes only -it does not correspond to any physical feature in the environment.

the first screen. Twelve stimulus locations, marked by identical wood pedestals $51 \mathrm{~cm}$ high, were positioned so that their spatial configurations were the same in regions $A$ and $B$. For the purpose of describing the experimental procedure and results, we shall treat the environment as having four quadrants-two formed by the physical division of region $A$ and two by an equivalent (yet imaginary) division of region $\mathbf{B}$. The imaginary division of region $\mathbf{B}$ is depicted in Figure 1 by a dotted line.

Location names. The learning phase of the experiment was described to subjects as being analogous to learning the locations and names of buildings on a university campus. Accordingly, 12 surnames were selected to meet the following criteria: (1) all had two syllables and approximately the same length; (2) each name began with a different letter of the alphabet; (3) no two names rhymed; (4) all names were of uniform ethnicity; (5) none were names of buildings actually on campus; (6) none were names of prominent figures known to subjects. A different random assignment of names to locations was used for each subject. The names were printed on pieces of paper in letters 1 -in. high and placed face down on the tops of the pedestals described above

Acquisition Procedure. Subjects participated individually. Before entering the environment, the subject was told that the purpose of the study was to find out more about how people learn new environments. It was explained that the subject's task would be to learn where everything in the experimental environment was "in relation to everything else in there." The subject was then led into the environment and positioned at a predetermined starting location near one of the pedestals. Equal numbers of subjects were assigned to starting points within each of the four quadrants of the environment. The starting stimulus location within the starting quadrant was determined randomly for each subject.

The subjects learned the environment by traveling from location to location in different random sequences. The experimenter escorted the subject to each location, whereupon the subject guessed the name associated with the location. The subject then turned over the name tag, noted the correct name, and returned it to its facedown position. On the very first trial, the subject did not have to guess the name and instead simply turned over the tag and noted the name. The trials continued until the subject correctly guessed all 12 location names twice in a row $(M=3.9$, range $=2-6)$.

The sequence of stimulus locations on the initial exposure and guessing trials was randomly determined, with the constraint that each successive location was in a different quadrant of the environment. Temporal and spatial contiguity are independent in this procedure, in the sense that all unvisited locations in the remaining three quadrants have an equal probability of being next in the sequence, regardless of their distance from the current stimulus. This includes locations that might be closer than other objects in the same quadrant as a given stimulus (e.g., those just on the other side of a barrier) as well as those farther away. The average shortest and longest distances in Experiment 1 between successive locations (computed across subjects and trials) were $143 \pm 7.96 \mathrm{~cm}$ and $408 \pm 12.46 \mathrm{~cm}$, respectively

The starting point for each trial was chosen randomly, with the constraint that it be in a quadrant different from that of the starting or ending point of the preceding trial

Test Procedure. Following the learning phase, the subjects were taken to another room, where they completed a recognition and free recall test for the location names. The recognition list contained 48 items: the 12 location names and 12 foils (selected according to the same criteria as those for the location names), each presented twice. Each location name appeared in one of six critical prime/target pairs. The pairs differed in the distance separating members (approximately 220 vs. $104 \mathrm{~cm}$ for far and close pairs) and in the regional relation of the members: A1/A2 pairs (each member from a different subarea of $A$ ), $A / B$ pairs (one member from $A$ and one from B), and B/B pairs (both members from B, selected to match the relative positions of $\mathrm{A} 1 / \mathrm{A} 2$ pairs). Within each distance category, pairs were selected so that the functional distance (i.e., the shortest travel distance) was the same across region relation categories. Thus, even though no barrier separated members of $B / B$ pairs, the travel distance between them was the same as that for $A / B$ and A1/A2 pairs. The one exception to this was the close A1/A2 combination, which was somewhat greater in travel distance than were its $A / B$ and $B / B$ counterparts.

If the trip trial method was successful in reducing the association between temporal and spatial contiguity, the temporal separation between visits to members of close and far critical pairs should have been comparable. As a check on this, we computed the average number of intervening locations between members of the prime/ target pairs across each subject's learning trials. The mean for close pairs $(3.37 \pm .15)$ did not differ reliably from the mean for far pairs (3.36 \pm .15$)\left[F(1,15)<1, M S_{e}=.67\right]$. The temporal separation for the A1/A2 $(3.52 \pm .23), \mathrm{A} / \mathrm{B} \quad(3.37 \pm .12)$, and $\mathrm{B} 1 / \mathrm{B} 2$ $(3.19 \pm .20)$ pair categories also did not differ significantly $\left[F(2,30)<1, M S_{e}=1.53\right]$, nor was the interaction between close/far and region combination significant $\left[F(2,30)<1, M S_{e}=\right.$ 1.15].

In the recognition test, the designation of the location to be prime or target was random and was reversed in the second presentation of the pair. The foils and prime/target pairs were ordered randomly with the constraint that at least one foil preceded every critical pair, and that the second presentation of an item did not occur until all items had been presented once. Two random orderings were used, with equal numbers of subjects assigned to each. The recognition items were displayed one at time on a computer display screen. The subjects were instructed to decide as quickly, yet accurately, as possible whether the name on the screen was that of one of the locations in the experimental environment. The subjects indicated their responses by pressing a "yes" or "no" button on a response pad. The left or right position of the "yes" and "no" response buttons was counterbalanced across starting position during the learning phase and sex of subject. A response terminated the display, and the next stimulus name appeared $250 \mathrm{msec}$ later.

In the free recall test, subjects were simply instructed to write down, in any order, the names of locations that had been in the experimental environment.

\section{Results}

Recognition latencies. Each subject's latencies for responses to targets within each prime/target type were averaged together. Only latencies for pairs in which both 
the target and prime were recognized correctly were included. Error rates were low (.02) and uniform across pair categories. Mean latencies for the prime/target pairs are shown in Table 1. Analysis of the latency data indicated no significant effect of the distance between primes and targets $\left[F(1,15)<1, M S_{\mathrm{e}}=6,036\right]$ or of the regional relation between them $\left[F(2,30)<1, M S_{c}=1,766\right]$, nor was there a significant interaction between distance and regional relation $\left[F(2,30)=1.98, M S_{e}=24,080\right]$.

Recall protocols. All subjects recalled the location names perfectly, allowing for misspellings and slight distortions. We examined the subjects' recall protocols for evidence of spatial organization in two ways. First, we tallied the number of subjects who clustered the location names by subarea, and we also noted the distance relationship between adjacent locations in the recall lists from different subareas. Unlike the recognition priming results, the recall protocols showed effects of both distance and region relations. For example, nearly all subjects $(94 \%)$ recalled the region $A$ names together as one cluster and the region $B$ names together as another. Within region $A$, $100 \%$ recalled the $A 1$ and $A 2$ names as separate clusters. When the recall sequence moved between $A 1$ and $A 2$, there was a moderate tendency ( $40 \%$ ) for the next item to be closest in distance. This tendency was less $(25 \%)$ when recall switched between regions A and B, but there was a fairly strong tendency ( $63 \%$ ) for the next name to be from the closest quadrant.

In a second approach to examining the recall data, we computed the average number of intervening items in each subject's protocol between members of the same six critical pair categories that were used in the recognition priming task. These data were then analyzed in the same manner as were the latency measures. The means for the recall separation measure are shown in Table 1. Recall separation was influenced by both distance and regional relations between items. The number of intervening items was significantly smaller for close pairs than for far pairs $\left[F(1,15)=15.27, M S_{\mathrm{e}}=.65, p<.01\right]$. A region effect was also obtained $\left[F(2,30)=7.81, M S_{\mathrm{e}}=4.09, p<\right.$ $.01]$, in which pairs within region $B$ were recalled closer

Table 1

Mean Recognition Latencies (in Milliseconds) and Recall Separation Measures for Prime/Target Pairs in Experiment 1 (with Standard Errors)

\begin{tabular}{|c|c|c|c|c|c|c|c|c|}
\hline \multirow[b]{3}{*}{ Distance } & \multicolumn{6}{|c|}{ Region Relation } & & \\
\hline & \multicolumn{2}{|c|}{ A1/A2 } & \multicolumn{2}{|c|}{ A/B } & \multicolumn{2}{|c|}{ B/B } & \multicolumn{2}{|c|}{ Combined } \\
\hline & $M$ & $S E$ & $M$ & $S E$ & $M$ & $S E$ & $M$ & $S E$ \\
\hline \multicolumn{9}{|c|}{ Latencies } \\
\hline $\begin{array}{l}\text { Far } \\
\text { Close } \\
\text { Combined }\end{array}$ & $\begin{array}{l}516 \\
611 \\
564\end{array}$ & $\begin{array}{l}26 \\
79 \\
42\end{array}$ & $\begin{array}{l}558 \\
500 \\
529\end{array}$ & $\begin{array}{l}42 \\
25 \\
25\end{array}$ & $\begin{array}{l}524 \\
522 \\
523\end{array}$ & $\begin{array}{l}24 \\
33 \\
20\end{array}$ & $\begin{array}{l}533 \\
545\end{array}$ & $\begin{array}{l}18 \\
30\end{array}$ \\
\hline \multicolumn{9}{|c|}{ Recall Separation } \\
\hline $\begin{array}{l}\text { Far } \\
\text { Close } \\
\text { Combined }\end{array}$ & $\begin{array}{l}2.40 \\
2.16 \\
2.27\end{array}$ & $\begin{array}{l}.37 \\
.52 \\
.32\end{array}$ & $\begin{array}{l}3.30 \\
2.81 \\
3.05\end{array}$ & $\begin{array}{l}.39 \\
.41 \\
.28\end{array}$ & $\begin{array}{r}1.68 \\
.47 \\
1.07\end{array}$ & $\begin{array}{l}.15 \\
.09 \\
.14\end{array}$ & $\begin{array}{l}2.46 \\
1.81\end{array}$ & $\begin{array}{l}.21 \\
.26\end{array}$ \\
\hline
\end{tabular}

together than $\mathrm{A} / \mathrm{B}$ pairs $[F(1,30)=7.67, \mathrm{p}<.01]$. A1/A2 pairs were intermediate in recall separation and did not differ significantly from either the $\mathrm{B} / \mathrm{B}$ or the $\mathrm{A} / \mathrm{B}$ pairs $(F \mathrm{~s}=2.82,1.19)$. Finally, a marginally significant distance $\times$ region interaction was obtained $[F(2,30)=$ $\left.3.11, M S_{\mathrm{e}}=.65, p<.06\right]$. The simple effect of distance was significant for the $\mathrm{B} / \mathrm{B}$ pairs $\left[F(1,15)=77.64, M S_{\mathrm{e}}\right.$ $=.15, p<.001]$, but not for the $\mathrm{A} / \mathrm{B}$ or $\mathrm{A} 1 / \mathrm{A} 2$ pairs $\left(F_{\mathrm{s}}=1.77,<1\right)$.

\section{Discussion}

The recognition priming results of Experiment 1 are clearly at odds with both our own previous research in which we used the same experimental materials, and with the research of other investigators who have consistently obtained spatial priming effects in recognition memory for experimentally learned configurations. In the present experiment, the subjects were able to recognize the names of locations in the configuration accurately without activating spatial relations between them, just as in everyday environments. Reducing the experimental coincidence between spatial proximity and temporal contiguity in rehearsal eliminated priming effects, thereby supporting the possibility that earlier reported effects were not, in fact, due solely to encoded spatial relations.

The spatial organization of subjects' recall protocols suggests that spatial relations were indeed encoded, and that they influenced retrieval during the recall task. Because subjects never traveled between locations in the orders or groupings represented in their recall protocols, these spatial relations were necessarily independent of the temporal contiguity in moving from one location to the next. Although the recall task did not explicitly require a locational decision, it did present the subject with the goal of retrieving an entire set of items. The set in the present case would be defined primarily by the spatial associations among elements. In the recognition task, however, the episodic familiarity of the location names relative to the foils would be sufficient for a correct response, and the learning procedure used here ensured that this familiarity was uniform for all locations and was not confounded with spatial relations.

One criticism of Experiment 1 is that subjects' recall protocols provide only indirect evidence that spatial relations were fully encoded. Thus, it is possible that spatial priming effects did not appear because spatial relations were only partially or weakly represented in subjects' memories for the configuration, as compared with the results of previous studies. One reason this might happen is that by keeping the location names hidden from view while the subject moved through the layout, we made rehearsal of spatial relations more difficult than learning methods that have produced priming effects. In Experiment 2, we modified the trip trial procedure to explore this possibility, and we obtained direct measures of the accuracy with which subjects encoded spatial relations. In addition, the encoding accuracy and priming effects for the modified trip trial learning method were compared 
with those produced by the procedure used in most previously published studies of spatial priming.

\section{EXPERIMENT 2}

\section{Method}

Subjects. Thirty-six subjects, 18 men and 18 women, participated in return for research credit in introductory psychology courses. None had served in Experiment 1. Equal numbers participated in the two learning conditions.

Stimulus materials. The experimental configuration and location names were the same as in Experiment 1.

Acquisition Procedure. The initial introductory phase of the learning procedure was identical to that in Experiment 1, and it was the same for the two learning conditions.

Free study condition. The free study condition was designed to parallel the essential features of the methodology used in previous experiments in which spatial priming effects for recognition memory have been reported (e.g., McNamara, 1986). Each study/test trial began with the location names face up on the pedestals. The subject studied the configuration for $2 \mathrm{~min}$, moving about the environment at will, and then faced away from the layout as the experimenter turned the name tags face down. The experimenter then gave the subject a shallow box containing a randomly ordered set of duplicate name tags with the instruction to place them on their correct pedestals. After placing all the duplicate tags, the subject turned over the originals to determine if his or her guesses had been correct, and returned the duplicates to the experimenter. The study/test trials continued until the subject correctly placed all of the duplicate names twice in a row $(M=2.67$, range $=2-5)$. During the study and test phases, the experimenter noted the subject's movement patterns and the manner in which the subject placed the duplicate names.

Trip trial condition. The trip trial procedure was the same as that in Experiment 1 with one change. After guessing the name associated with a location, the subject then turned over the name tag, leaving it exposed for the duration of the trial. Thus, when the subject reentered a region, at least one name was already visible, and by the end of a trial, all names were face up. On the very first trial, the subject did not have to guess the name and instead simply turned over the tag and noted the name. After all 12 locations had been visited, the subject faced away from the configuration while the experimenter returned the name tags to their face-down position for the next trial. As in Experiment 1, the trials continued until the subject correctly guessed all 12 location names twice in a row $(M=$ 3.5 , range $=2-5$ ). The average shortest and longest distances in Experiment 2 between successive locations (computed across subjects and trials) were $147 \pm 3.86 \mathrm{~cm}$ and $434 \pm 4.67 \mathrm{~cm}$.

Test Procedure. The subjects first completed a recognition test with the same materials and procedure as those in Experiment 1, and then were given a free recall test for the location names. Next, subjects made magnitude estimates of the distances between the locations in the critical pairs by placing an " $X$ " along a $10-\mathrm{cm}$ line. The experimenter explained that the line represented the same distance as the length of one of the outside walls of the experimental room, and that the distance to be estimated was straight-line or "crow-flight" distance. The order of the pairs was random. Finally, subjects produced sketch maps of the environment by drawing solid lines to indicate the position of the barriers, placing " $\mathrm{Xs}$ " to represent locations of the pedestals, then labeling each location.

\section{Results}

Temporal/spatial contiguity. For trip trial subjects, the mean number of intervening items between members of close $(3.26 \pm .19)$ and far $(3.35 \pm .17)$ prime/target pairs did not differ reliably $\left[F(1,17)<1, M S_{\mathrm{e}}=1.17\right]$, as in Experiment 1. The separations between A1/A2 $(3.27 \pm .16), \mathrm{A} / \mathrm{B}(3.73 \pm .23)$, and $\mathrm{B} / \mathrm{B}(2.91 \pm .25)$ pairs were also comparable $\left[F(2,34)=2.49, M S_{\mathrm{e}}=2.44\right]$, and the interaction between distance and region type was not significant $\left[F(2,34)<1, M S_{\mathrm{e}}=1.64\right]$.

Although direct measures of temporal contiguity were not available for free study subjects, informal observations of their behavior during the learning phase confirmed our speculation concerning their encoding and rehearsal strategies. All studied the locations region by region. Within a given region, they scanned back and forth between adjacent locations. During the test phase, all subjects chose to position themselves in a subarea and select name tags from the box of duplicates for spatially proximate locations in that region.

Recognition latencies. The mean recognition latencies for each condition are shown in Table 2. Only latencies for pairs in which both the target and the prime were recognized correctly were included. Error rates were low $(.05)$ and uniform across categories and across learning conditions.

An overall analysis of the latencies indicated significant effects of the distance between primes and targets $\left[F(1,34)=5.83, M S_{\mathrm{e}}=11,987, p<.05\right]$ and of the regional relation between them $\left[F(2,68)=3.28, M S_{\mathrm{e}}=\right.$ $16,182, p<.05]$, and no significant interaction between the two variables $[F(2,68)<1]$. Both the distance and the region effects, however, were reliable only for the free study subjects. ${ }^{2}$ Free study subjects responded more quickly to targets preceded by close primes than to those preceded by far primes $\left[F(1,17)=4.50, M S_{\epsilon}=16,294\right.$, $p<.05]$. Latencies for $\mathrm{A} 1 / \mathrm{A} 2$ and $\mathrm{B} / \mathrm{B}$ pairs, which did not differ significantly from each other, were together significantly faster than latencies for the $\mathrm{A} / \mathrm{B}$ pairs $[F(1,34)$ $\left.=4.76, M S_{e}=12,895, p<.05\right]$. The interaction between distance and region was not significant for the free study subjects $\left[F(2,34)<1, M S_{\mathrm{e}}=11,423\right]$. In the trip trial learning condition, the effects of distance $(F=1.38$, $\left.M S_{\mathrm{e}}=7,681\right)$, region $\left(F<1, M S_{\mathrm{e}}=19,470\right)$, and their interaction $\left(F<1, M S_{\mathrm{e}}=16,791\right)$ were all nonsignificant, as in Experiment 1.

Table 2

Mean Recognition Latencies (with Standard Errors) for the Free Study and Trip Trial Conditions in Experiment 2

\begin{tabular}{|c|c|c|c|c|c|c|c|c|}
\hline \multirow[b]{3}{*}{ Distance } & \multicolumn{6}{|c|}{ Region Relation } & & \\
\hline & \multicolumn{2}{|c|}{$\mathrm{A} 1 / \mathrm{A} 2$} & \multicolumn{2}{|c|}{ A/B } & \multicolumn{2}{|c|}{ B/B } & \multicolumn{2}{|c|}{ Combined } \\
\hline & $M$ & $S E$ & $M$ & $S E$ & $M$ & $S E$ & $M$ & $S E$ \\
\hline \multicolumn{9}{|c|}{ Free Study Condition } \\
\hline Far & 586 & 41 & 655 & 44 & 559 & 36 & 600 & 24 \\
\hline Close & 570 & 32 & 574 & 31 & 499 & 27 & 548 & 18 \\
\hline Combined & 578 & 25 & 615 & 27 & 529 & 23 & & \\
\hline \multicolumn{9}{|c|}{ Trip Trial Condition } \\
\hline Far & 574 & 55 & 593 & 35 & 569 & 28 & 579 & 23 \\
\hline Close & 553 & 28 & 579 & 33 & 544 & 16 & 560 & 15 \\
\hline Combined & 564 & 31 & 586 & 24 & 557 & 16 & & \\
\hline
\end{tabular}

Note-Times are given in milliseconds. 
Recall protocols. All subjects in both conditions correctly recalled the location names, allowing for misspellings and slight distortions. Despite the recognition priming differences between learning conditions noted above, the spatial organization of the recall protocols was similar in the two conditions. For example, nearly all subjects in both groups recalled all the region $A$ names and region $B$ names together (free study $=94 \%$, trip trial = $83 \%$ ). Within region $A, 94 \%$ of both groups recalled the $A 1$ and $A 2$ names as separate clusters. When the recall sequence moved between $A 1$ and $A 2$, there was a moderate tendency for the next item to be closest in distance (free study $=61 \%$, trip trial $=60 \%$ ). This tendency was less when recall switched between regions $A$ and $B$ (free study $=55 \%$, trip trial $=47 \%$ ), but there was a strong tendency in both groups for the next name to be from the closest quadrant (free study $=75 \%$, trip trial $=81 \%$ ).

As in Experiment 1, we computed the average number of intervening items in each subject's recall protocol between members of the same six pair categories that were used in the recognition priming task. Means for the prime/target combinations in each condition are shown in Table 3. The pattern of distance and region organization in the protocols was very similar to those in Experiment 1 , in both the trip trial and the free study conditions. An overall analysis indicated that recall separation between items was significantly smaller for close pairs than for far pairs $\left[F(1,34)=48.19, M S_{\mathrm{e}}=.73, p<.001\right]$. A region effect was also obtained $\left[F(2,68)=9.56, M S_{\text {c }}\right.$ $=3.56, p<.001]$, in which pairs within region $\mathrm{B}$ were recalled closer together than both $\mathrm{A} / \mathrm{B}$ pairs $[F(1,68)=$ $8.29, p<.01]$ and $\mathrm{A} 1 / \mathrm{A} 2$ pairs $[F(1,68)=6.00, p<$ $.05]$. The $A 1 / A 2$ and $A / B$ pairs did not differ significantly $(F<1)$. Finally, a significant distance $\times$ region interaction was obtained $\left[F(2,68)=3.72, M S_{\mathrm{e}}=.48\right.$, $p<.05]$. The distance effect was greatest in the $\mathrm{B} / \mathrm{B}$ pairs, as in Experiment 1, but here it was significant for the other types of region relations as well.

Neither the main effect of learning condition nor its interactions with distance and region were significant (maximum $F=2.68$ ). However, unlike the recognition priming analyses, the overall pattern of recall results held equally well for each of the two learning groups, with

Table 3

Mean Recall Separation Between Prime/Target Pairs in Experiment 2 (with Standard Errors)

\begin{tabular}{|c|c|c|c|c|c|c|c|c|}
\hline \multirow[b]{3}{*}{ Distance } & \multicolumn{6}{|c|}{ Region Relation } & & \\
\hline & \multicolumn{2}{|c|}{$\mathrm{Al} / \mathrm{A} 2$} & \multicolumn{2}{|c|}{ A/B } & \multicolumn{2}{|c|}{$\mathrm{B} / \mathrm{B}$} & \multicolumn{2}{|c|}{ Combined } \\
\hline & $M$ & $S E$ & $M$ & $S E$ & $M$ & $S E$ & $M$ & $S E$ \\
\hline \multicolumn{9}{|c|}{ Free Study Condition } \\
\hline $\begin{array}{l}\text { Far } \\
\text { Close } \\
\text { Combined }\end{array}$ & $\begin{array}{l}2.07 \\
1.41 \\
1.74\end{array}$ & $\begin{array}{l}.07 \\
.20 \\
.12\end{array}$ & $\begin{array}{l}2.83 \\
1.94 \\
2.39\end{array}$ & $\begin{array}{l}.26 \\
.43 \\
.26\end{array}$ & $\begin{array}{r}1.76 \\
.78 \\
1.27\end{array}$ & $\begin{array}{l}.16 \\
.13 \\
.13\end{array}$ & $\begin{array}{l}2.22 \\
1.38\end{array}$ & $\begin{array}{l}.12 \\
.17\end{array}$ \\
\hline \multicolumn{9}{|c|}{ Trip Trial Condition } \\
\hline $\begin{array}{l}\text { Far } \\
\text { Close } \\
\text { Combined }\end{array}$ & $\begin{array}{l}2.93 \\
2.22 \\
2.57\end{array}$ & $\begin{array}{l}.27 \\
.47 \\
.27\end{array}$ & $\begin{array}{l}3.00 \\
2.66 \\
2.83\end{array}$ & $\begin{array}{l}.54 \\
.60 \\
.40\end{array}$ & $\begin{array}{r}1.78 \\
.50 \\
1.14\end{array}$ & $\begin{array}{l}.11 \\
.08 \\
.13\end{array}$ & $\begin{array}{l}2.56 \\
1.79\end{array}$ & $\begin{array}{l}.22 \\
.28\end{array}$ \\
\hline
\end{tabular}

just one exception. The simple interaction between distance and region was significant for the trip trial subjects $\left[F(2,34)=3.98, M S_{e}=.50, p<.03\right]$, but not for those in the free study condition $\left[F(2,34)<1, M S_{\mathrm{e}}=.46\right]$. For trip trial subjects, the simple effect of distance was significant for the $\mathrm{B} / \mathrm{B}$ pairs $\left[F(1,17)=72.76, M S_{\mathrm{e}}=\right.$ $.20, p<.001]$ and the $\mathrm{A} 1 / \mathrm{A} 2$ pairs $[F(1,17)=4.71$, $\left.M S_{\mathrm{e}}=.95, p<.05\right]$, but not for the A/B pairs $[F(1,17)$ $\left.=1.76, M S_{\mathrm{e}}=.60\right]$.

Encoding accuracy. An informal examination of subjects' sketch maps suggested that participants in both learning conditions were fairly accurate in encoding the experimental configuration. All subjects correctly labeled the stimulus locations in their maps and placed all locations in the correct regions. The relative positions of the stimulus locations were also fairly accurate, with the exception that about one third of the subjects in both conditions noticeably displaced the locations in region $B$ with respect to their counterparts in region A.

More specific information about encoding accuracy was obtained by examining subjects' estimates of distance between members of critical pairs. The average correlation between the estimates and actual distances was high and nearly the same for both the trip trial $(.72 \pm .05)$ and free study $(.69 \pm .06)$ conditions. Thus, it is not likely that the difference between the two conditions in distance priming effects was due to the failure of trip trial subjects to encode distances or to greater distortions in their representations of distance. An analysis of subjects' mean errors in estimating distance between members of critical pairs showed no main effect of learning condition $(F<1)$, nor any interactions between condition and the other independent variables (maximum $F=1.43$ ). The only reliable result was an overall effect of region relation $[F(2,68)=$ 9.13, $\left.M S_{\mathrm{e}}=1,264, p<.001\right]$. Distances for B/B pairs were underestimated (mean error $=-8.67 \mathrm{in}$.) compared to distances for $\mathrm{A} / \mathrm{B}$ pairs (mean error $=+14.62$ in.) $[F(1,68)=7.66, p<.01]$ and for A $1 / \mathrm{A} 2$ pairs (mean error $=+11.57$ in.) $[F(1,68)=5.84, p<.05]$, which did not differ significantly from each other $(F<1)$. The same pattern held for each learning condition analyzed separately.

\section{Discussion}

Despite the change in the trip trial learning procedure for Experiment 2, it again failed to produce recognition priming effects. The free study method, patterned after the procedure used in other studies of experimental arrays, was associated with both distance and region priming effects. Taken together, the results of these two studies indicate that spatial priming in recognition memory depends on how environments are learned.

The distance estimates and sketch maps in Experiment 2 show that subjects in the trip trial condition possessed knowledge of spatial relations comparable in accuracy to the knowledge of free study subjects, and that this knowledge influenced their retrieval of object identities in a manner similar to that for the free study subjects when 
the task (i.e., free recall) required knowledge of the objects as a related set.

Our observations of subjects' behaviors during the study and test trials of the free study learning sessions are consistent with the possibility that for these subjects temporal and spatial contiguity were highly associated in encoding and rehearsing the experimental layout. These behaviors seem very likely to have produced strong episodic links between adjacent objects within regions that can account for the priming results. This is a tentative conclusion, however, because of the informal nature of our observations. One way a stronger case could be made would be to modify the trip trial procedure so that temporal and spatial distance coincided. Obtaining spatial priming effects in such a modification would rule out the possibility that the lack of priming effects in the trip trial method was due to some peculiarity of the procedure and would more directly implicate the temporal/spatial nature of encoding and rehearsal in the free study technique as being responsible for spatial priming.

\section{EXPERIMENT 3}

Our goal in Experiment 3 was to examine spatial priming effects in a modified version of the trip trial method in which temporal and spatial contiguity were highly associated in subjects' learning experiences.

\footnotetext{
Method

Subjects. Sixteen subjects ( 8 women and 8 men) participated in return for research credit in introductory psychology courses. None had served in Experiments 1 or 2 . Experiment 3 was the same as the first two experiments in all aspects apart from the learning procedure.

Acquisition Procedure. Temporal and spatial separation might coincide during trips through an environmental configuration when the paths followed are functionally optimal-that is, when the next location visited is closest in distance, allowing for physical constraints imposed by barriers to locomotion. In Experiment 3, subjects traveled from location to location along such an optimal path through the same configuration as that used in Experiments 1 and 2 , guessing the name at each location as in the trip trials of the first two studies.

The path connected the 12 locations in roughly a circular pattern, passing through each location once, with comparable locations connected in regions $\mathrm{A}$ and $\mathrm{B}$. When the $\mathrm{A} / \mathrm{B}$ and $\mathrm{A} 1 / \mathrm{A} 2$ barriers were crossed, the next location visited was the closest to the preceding location. Each trial began at a different starting point, and the direction of travel was reversed from that on the preceding trial. Four starting points were used, one in each of the quadrants of the layout, and these were repeated if the subject required more trials to reach criterion. The subjects traveled the remainder of the path in one region before continuing to the next. ${ }^{3}$ The average number of intervening locations between members of pairs in the close $(104-\mathrm{cm})$ and far $(220-\mathrm{cm})$ distance categories was $.40 \pm .24$ and $3.50 \pm .82$, respectively.

Learning trials continued until the subjects correctly identified all locations on the path twice in a row $(M=4.7$, range $=3-7)$. Following the learning phase, the subjects completed the same recognition, recall, distance estimation, and map drawing tasks as in Experiment 2.
}

\section{Results}

Mean recognition latencies and recall separation scores for the prime/target combinations are shown in Table 4 .

Recognition latencies. Each subject's latencies for responses to targets within each prime/target type were averaged together. Only latencies for pairs in which both the target and the prime were recognized correctly were included. Error rates were low (.04) and uniform across categories. The subjects recognized targets preceded by close primes more quickly than targets preceded by far primes, as in the free study condition of Experiment 2 $\left[F(1,15)=4.65, M S_{\mathrm{e}}=5,120, p<.05\right]$. The spatial priming effect here tended to be greater for $B / B$ pairs than for $A / B$ or $A 1 / A 2$ pairs, though the interaction between distance and region was not significant $[F(2,30)=2.11$, $\left.M S_{\mathrm{e}}=4,418, p<.14\right]$, as in Experiment 2. Latencies did not differ significantly across region combinations $[F(2,30)<1]$.

Recall protocols. The structure of subjects' recall protocols was similar to that observed in Experiments 1 and 2. Nearly all subjects recalled all the region $A$ names and region $B$ names together $(81 \%)$. When the recall sequence moved between $A$ and $B$, there was a strong tendency ( $70 \%$ ) for the next name to be from the closest quadrant. Within region $A, 94 \%$ recalled the $A 1$ and $A 2$ names as separate clusters. For the present subjects, this pattern is similar to the nature of the path followed during the learning trials. However, despite the optimal nature of the path, there was again only a moderate tendency for the next item to be closest in distance when recall switched between A1 and A2 (50\%) and between A and B (53\%).

Analysis of the recall separation scores for prime/target pairs showed reliable effects of both distance $[F(1,15)=$ $\left.5.18, M S_{\mathrm{e}}=.91, p<.05\right]$ and regional relation $[F(2,30)$ $\left.=5.77, M S_{\mathrm{c}}=3.38, p<.01\right]$. As in Experiments 1 and 2 , the mean separation was smaller for close pairs than for far pairs. B/B pairs were recalled more closely than $\mathrm{A} / \mathrm{B}$ pairs $[F(1,30)=5.69, p<.05]$ and $\mathrm{A} 1 / \mathrm{A} 2$ pairs, though the latter contrast was not significant here $[F(1,30)<1]$. As was the case with the free study sub-

Table 4

Mean Recognition Latencies (in Milliseconds) and Recall Separation Measures for Prime/Target Pairs in Experiment 3 (with Standard Errors)

\begin{tabular}{|c|c|c|c|c|c|c|c|c|}
\hline \multirow[b]{3}{*}{ Distance } & \multicolumn{6}{|c|}{ Region Relation } & & \\
\hline & \multicolumn{2}{|c|}{$\mathrm{A} 1 / \mathrm{A} 2$} & \multicolumn{2}{|c|}{ A/B } & \multicolumn{2}{|c|}{ B/B } & \multicolumn{2}{|c|}{ Combined } \\
\hline & $M$ & $S E$ & $M$ & $S E$ & $M$ & $S E$ & $M$ & $S E$ \\
\hline \multicolumn{9}{|c|}{ Latencies } \\
\hline Far & 522 & 23 & 530 & 30 & 538 & 27 & 530 & 15 \\
\hline Close & 514 & 25 & 513 & 28 & 468 & 20 & 498 & 14 \\
\hline Combined & 518 & 17 & 521 & 20 & 503 & 18 & & \\
\hline \multicolumn{9}{|c|}{ Recall Separation } \\
\hline Far & 2.33 & .31 & 3.16 & .40 & 2.00 & .06 & 2.50 & .18 \\
\hline Close & 1.97 & .39 & 3.06 & .38 & 1.13 & .44 & 2.05 & .26 \\
\hline Combined & 2.15 & .25 & 3.11 & .27 & 1.56 & .24 & & \\
\hline
\end{tabular}


jects in Experiment 2, the interaction between distance and region was not significant $\left[F(2,30)=1.86, M S_{\mathrm{e}}=.68\right]$.

Encoding accuracy. The average correlation between estimated and actual distance was $.67 \pm .10$. As in Experiment 2 , subjects underestimated distances for $B / B$ pairs (mean error $=-12.26$ in.) relative to the $\mathrm{A} 1 / \mathrm{A} 2$ and $\mathrm{A} / \mathrm{B}$ pairs (mean combined error $=+3.55 \mathrm{in}$.) $\left[F(1,30)=7.26, M S_{\mathrm{e}}=551, p<.05\right]$, which again did not differ reliably from each other $[F(1,30)<1]$. The subjects' sketch maps were comparable in accuracy to those produced in Experiment 2.

\section{Discussion}

Introducing an association between temporal and spatial separation during the trip trials produced a spatial priming effect in the recognition task. This result, in conjunction with our earlier research (Sherman, 1987) and the results of Experiments 1 and 2, shows that the trip trial method produces spatial priming when a temporal/spatial association is present, but that it does not do so when this association is reduced. The similarity in encoding accuracy and structure of recall protocols produced by the free study and different versions of the trip trial conditions suggests that differences in the recognition priming effect are probably not simply due to differences in how accurately spatial relations are represented in subjects' memories for the environmental information.

We have argued that the trip trial method is similar to the learning of natural environments, in which rehearsal contiguity and spatial relations are often independent. As noted earlier, spatial priming does not occur for naturally learned environments unless the task requires a spatial decision. Our purpose in Experiment 4 was to determine whether this would also be true of an experimental environment learned by the original random-sequence trip trial method.

\section{EXPERIMENT 4}

\footnotetext{
Method

Twenty-three subjects (12 women and 11 men) participated in return for research credit in introductory psychology courses. None had served in Experiments 1-3. All aspects of Experiment 4 were identical to the random-sequence trip trial condition of Experiment 2, with the exception of the priming task.

The location-decision task was patterned after one used in a recent study by McNamara, Altarriba, et al. (1989), which yielded spatial priming in both a naturally learned environment (a college campus) and an experimentally learned configuration (a novel map). In the present experiment, the subjects were presented with a location name on a computer display and instructed to decide, as quickly yet accurately as possible, whether the name was in region $A$ or in region $\mathrm{B}$, and to press an appropriately labeled button on a response pad to indicate their decision. If the name was a foil, the subjects were instructed to press one button if the name began with one of the letters A-O and another button if it began with one of the letters P-Z. The lists of foils and prime/target pairs were constructed in the same manner as in Experiments 1-3.

Following the location-decision task, the subjects completed the same free recall, distance estimation, and map drawing tasks as in Experiments 2 and 3.
}

The mean number of learning trials to criterion was 3.7 (range $=2-7$ ). The average shortest and longest distances between locations were $155.28 \pm 4.06 \mathrm{~cm}$ and $440.10 \pm 3.63 \mathrm{~cm}$, respectively. The number of intervening locations did not differ reliably between members of close $(3.18 \pm .16)$ and far $(3.39 \pm .15)$ critical pairs $\left[F(1,22)=1.43, M S_{c}=1.07\right]$ or between members of $\mathrm{B} / \mathrm{B}$ $(3.49 \pm .20), \mathrm{A} 1 / \mathrm{A} 2(3.35 \pm .19)$, and $\mathrm{A} / \mathrm{B}(3.03 \pm .17)$ pairs $\left[F(2,44)=1.48, M S_{e}=1.69\right]$. The interaction between distance and region was not significant $[F(2,44)<1]$.

\section{Results}

Mean decision latencies and recall separation scores are presented in Table 5.

Latency data. Error rates for the location decisions were very low (.01) and uniform across prime/target categories. Analysis of the latencies showed significant effects for distance between primes and targets $[F(1,22)$ $\left.=8.33, M S_{\mathrm{e}}=157,766, p<.01\right]$, and for their regional relation $\left[F(2,44)=3.40, M S_{\mathrm{e}}=211,034, p<.05\right]$. The interaction between distance and region was not significant $(F<1)$. The subjects located a target more quickly when it was preceded by a close prime than when it was preceded by a far prime. Targets in $B / B$ pairs were located more quickly than targets in $\mathrm{A} / \mathrm{B}$ pairs $[F(1,44)=$ $6.21, p<.05]$. Latencies for locating A1/A2 targets were marginally slower than they were for locating $B / B$ targets $[F(1,44)=3.66, p<.10]$, and they did not differ from latencies for locating $\mathrm{A} / \mathrm{B}$ targets $(F<1)$.

Recall protocols. As in Experiments 1-3, all subjects correctly recalled the location names, again allowing for misspellings and slight distortions. Recall protocols were structured similarly as well. Here, $96 \%$ of the subjects recalled locations in regions $A$ and $B$ together. Within region $A, 87 \%$ recalled names in $A 1$ and $A 2$ as separate clusters. When their recall sequence moved between $A 1$ and $A 2,70 \%$ of the subjects listed the closest name in the other region, and $66 \%$ did so when moving between regions $A$ and $B$. As before, when recall switched between regions $A$ and $B$, there was a strong tendency $(75 \%)$ for the next name to be in the closest quadrant.

Analysis of the recall separation scores showed the same effects of distance $\left[F(1,22)=64.50, M S_{\mathrm{c}}=.30\right.$, $p<.01]$ and region $\left[F(1,44)=12.58, M S_{\mathrm{e}}=3.17, p<\right.$

Table 5

Mean Location Decision Latencies (in Milliseconds) and

Recall Separation Measures for Prime/Target Pairs in Experiment 4 (with Standard Errors)

\begin{tabular}{|c|c|c|c|c|c|c|c|c|}
\hline \multirow[b]{3}{*}{ Distance } & \multicolumn{6}{|c|}{ Region Relation } & & \\
\hline & \multicolumn{2}{|c|}{$\mathrm{A} 1 / \mathrm{A} 2$} & \multicolumn{2}{|c|}{ A/B } & \multicolumn{2}{|c|}{ B/B } & \multicolumn{2}{|c|}{ Combined } \\
\hline & $M$ & $\overline{S E}$ & $M$ & $\overline{S E}$ & $M$ & $\overline{S E}$ & $M$ & $S E$ \\
\hline \multicolumn{9}{|c|}{ Latencies } \\
\hline $\begin{array}{l}\text { Far } \\
\text { Close } \\
\text { Combined }\end{array}$ & $\begin{array}{l}1,264 \\
1,078 \\
1,171\end{array}$ & $\begin{array}{r}135 \\
79 \\
79\end{array}$ & $\begin{array}{l}1,359 \\
1,095 \\
1,227\end{array}$ & $\begin{array}{r}126 \\
90 \\
79\end{array}$ & $\begin{array}{c}1,055 \\
921 \\
988\end{array}$ & $\begin{array}{l}94 \\
51 \\
54\end{array}$ & $\begin{array}{l}1,266 \\
1,031\end{array}$ & $\begin{array}{l}70 \\
44\end{array}$ \\
\hline \multicolumn{9}{|c|}{ Recall Separation } \\
\hline $\begin{array}{l}\text { FaI } \\
\text { Close } \\
\text { Combined }\end{array}$ & $\begin{array}{l}2.19 \\
1.28 \\
1.73\end{array}$ & $\begin{array}{l}.27 \\
.39 \\
.25\end{array}$ & $\begin{array}{l}3.13 \\
2.91 \\
3.02\end{array}$ & $\begin{array}{l}.32 \\
.26 \\
.21\end{array}$ & $\begin{array}{r}1.78 \\
.65 \\
1.22\end{array}$ & $\begin{array}{l}.11 \\
.13 \\
.12\end{array}$ & $\begin{array}{l}2.37 \\
1.62\end{array}$ & $\begin{array}{l}.16 \\
.20\end{array}$ \\
\hline
\end{tabular}


.01], as were found in Experiments 1-3. As shown in Table 5 , the mean separation was smaller for close pairs than it was for far pairs. B/B pairs were recalled more closely than were $\mathrm{A} / \mathrm{B}$ pairs $[F(1,44)=11.75, p<.01]$, as were $\mathrm{A} 1 / \mathrm{A} 2$ pairs $[F(1,44)=6.04, p<.05]$. The $\mathrm{B} / \mathrm{B}$ and A1/A2 pairs did not differ significantly $(F<1)$. An interaction between distance and region was also obtained $\left[F(2,44)=3.30, M S_{e}=.78, p<.05\right]$. The distance effect was significant for A1/A2 $(F=20.59, p<.001)$ and $\mathrm{B} / \mathrm{B}$ pairs $(F=31.12, p<.001)$, but not for A/B pairs $(F<1)$.

Encoding accuracy. The average correlation between estimated and actual distances was $.74 \pm .07$. The subjects underestimated distances for $B / B$ pairs (mean error $=-6.95$ in.) relative to $\mathrm{A} / \mathrm{B}$ pairs (mean error $=$ +7.31 in. $)\left[F(1,44)=2.56, M S_{\mathrm{e}}=916, p<.05\right]$. Mean estimation errors for $\mathrm{A} 1 / \mathrm{A} 2$ pairs (+5.44 in.) were again not significantly different from those for the A/B pairs $(F<1)$, but here they also did not differ from those for the $\mathrm{B} / \mathrm{B}$ pairs $(F=1.93)$. The subjects' sketch maps were comparable in accuracy to those produced in Experiments 2 and 3.

\section{Discussion}

The subjects who learned the environment with the random-sequence trip trial method showed spatial priming effects in a location-decision task (Experiment 4) but not in a simple recognition task (Experiments 1 and 2). This task-related difference in priming has previously been found only in studies of everyday environments.

These results, in combination with the recall data from Experiments 1,2, and 4, suggest that spatial relations are indeed encoded in situations in which the association between temporal and spatial contiguity is weak, but that these relations do not guide retrieval of environmental information unless the demands of the task have an explicit (location-decision task) or implicit (exhaustive recall task) spatial component.

\section{GENERAL DISCUSSION}

Our results call into question the interpretation of recognition priming in memory for experimentally learned environments as being due solely to encoded spatial relations. Rather, it seems likely that such effects can arise from associations formed from the rehearsal patterns encouraged by a particular method of learning the experimental layout. When rehearsal contiguity and spatial proximity are independent, as in the trip trial method used here, spatial priming in recognition does not occur. Converging results with a somewhat different procedure have been recently obtained by Clayton and Habibi (1991).

Our conclusion is, in some respects, similar to that proposed by Clayton and Chattin (1989). They suggest that learning new environmental information, whether in an experimental or natural context, results in an episodic memory structure in which the interconnections are formed from encoding and rehearsal strategies. These in- terconnections are likely to correspond to spatial relations, because adjacent locations are likely to be encoded and rehearsed together. A recognition task, they suggest, makes use primarily of the episodic structure, and therefore priming occurs in accordance with the episodic connections, which coincide with spatial relations. Our main disagreement with this analysis concerns the assumed generality of encoding and rehearsing adjacent locations together. This strategy seems to have been likely in the free study method used in most experimental contexts, and it may characterize some types of natural situations, such as the intentional study of geographic information from maps. However, much experience with everyday environments involves incidental encoding and integration of information accumulated from varied perspectives and multiple temporal associations between encounters with objects (Evans \& Pezdek, 1980; Siegel \& White, 1975). Furthermore, the structural elements in everyday environments frequently place constraints on vision and locomotion that reduce or even reverse the association between temporal and spatial separation, as in the case of objects on adjacent floors of a multistory building, or houses separated by natural geographic features that necessitate nonoptimal paths between them.

The data from the present experiments require modification of the restricted-context explanation of McNamara, Altarriba, et al. (1989) in two ways. First, we have shown that the experiencing of identities and locations only in the same spatial context is not sufficient to produce spatial priming in recognition. In the trip trial method used in Experiments 1 and 2, the subjects' only experience with the names occurred in the context of learning their positions in the experimental array, yet the subjects did not base their recognition decisions on spatial relations. Second, we do not doubt that objects in naturally learned environments may acquire multiple sources of familiarity, but our data suggest that the familiarity afforded by experiencing the objects through a variety of movement patterns and from a number of perspectives is adequate for making recognition decisions. It may be, then, that varied movement patterns within an environment produce nonspatial links among object identities that allow efficient decisions concerning membership in the array, and that these associations are either represented separately from spatial relations or integrated with them in some complex and as yet undetermined manner.

One of the implications of our results concerns the status of previous conclusions regarding the structure of environmental memory. Specifically, regional priming effects in recognition have been interpreted as evidence for hierarchical models of environmental memory (McNamara, 1986; McNamara, Hardy, \& Hirtle, 1989: Sherman, 1987). The general claim of these models is that objects in the same physically or subjectively defined subarea of a configuration are represented in the same branch of a graph-theoretic tree in which the degree of detail differs across the levels of the hierarchy. Recognition priming that is greater for objects in the same subarea than for 
objects in different subareas supports the hierarchical claim because encoding objects in different branches of the tree "creates an additional component to psychological distance" (McNamara, 1986, p. 96). As our data suggest, however, regional priming in recognition may be accounted for simply by strategic rehearsal patterns that create stronger links among objects in the same region, a decidedly nonhierarchical explanation.

Although recognition priming might be considered ambiguous support for hierarchical models, there are converging sources of evidence that are consistent with the models' general claims (see Hirtle \& Jonides, 1985; McNamara, Hardy, \& Hirtle, 1989; Stevens \& Coupe, 1978). In the present research, the trip trial recall data from all four experiments, the location-decision priming effects in Experiment 4, and the distance estimation errors in Experiments 2-4 revealed regional associations that suggest a hierarchical organization. In all four experiments, there was a general tendency to recall objects within a major region (B/B pairs) more closely together than objects in different major regions (A/B pairs), with objects in different subordinate areas of the same major region (A1/A2 pairs) intermediate. This same regional pattern was reflected in the priming effects obtained in Experiment 4, and it parallels the distance estimation errors observed in Experiments 2-4 in which subjects tended to underestimate distances within a major region $(B / B)$, and to overestimate functionally equivalent distances between objects in different major regions (A/B). Overestimations of distance between objects in different subordinate areas of the same major region (A1/A2) were again intermediate. These results cannot be accounted for by temporal contiguity in rehearsal, and they seem to pose difficulties for any simple nonhierarchical model of which we are aware.

In general, the data presented here add to the emerging view of environmental memory as a complex and multiply determined structure. Environmental information, it seems, can be accessed in a variety of ways, depending on the current evolutionary state of the memory structure containing it, the nature of the experiences through which it is acquired, and the nature of the task for which it is being retrieved. An appropriate guiding principle for future research in this area might be derived from the conclusion offered by Merrill and Baird (1987), who have argued that spatial proximity is only one of many factors that may determine the level of relatedness among environmental items, and that "spatial relations do not by themselves govern the organization of environmental memory"' (p. 107).

\section{REFERENCES}

Clayton, K. N., \& Chattin, D. (1989). Spatial and semantic priming effects in tests of spatial knowledge. Journal of Experimental Psychology: Learning. Memory \& Cognition, 15, 495-506.

Clayton, K. N., \& HaBiBi, A. (1991). Contribution of temporal contiguity to the spatial priming effect. Journal of Experimental Psychology: Learning, Memory, \& Cognition, 17, 263-271.
Evans, G. W. (1980). Environmental cognition. Psychological Bulletin, 88, 259-287.

Evans, G. W., Pezdek, K. (1980). Cognitive mapping: Knowledge of real-world distance and location information. Joumal of Experimental Psychology: Human Learning \& Memory, 6, 13-24.

HiRTLE, S. C., \&ONIDES, J. (1985). Evidence of hierarchies in cognitive maps. Memory \& Cognition, 13, 208-217.

MCNAmara, T. P. (1986). Mental representations of spatial relations. Cognitive Psychology, 18, 87-121.

McNamara, T. P., Altarriba, J., Bendele, M., Johnson, S. C., \& ClAYTON, K. N. (1989). Constraints on priming in spatial memory: Naturally learned versus experimentally learned environments. Memory \& Cognition, 17, 444-453.

McNamara, T. P., Hardy, J. K., \& Hirtle, S. C. (1989). Subjective hierarchies in spatial memory. Joumal of Experimental Psychology: Learning, Memory, \& Cognition, 15, 211-227.

McNamara, T. P., Ratcliff, R., McKoon, G. (1984). The mental representation of knowledge acquired from maps. Journal of $E_{x}$ perimental Psychology: Leaming, Memory, \& Cognition, 10, 723-732.

Merrill, A. A., \& Baird, J. C. (1987). Semantic and spatial factors in environmental memory. Memory \& Cognition, 15, 101-108.

SHERMAN, R. C. (1987, May). Hierarchical structure in cognitive maps. Paper presented at the meeting of the Midwestern Psychological Association, Chicago.

Sherman, R. C., Croxton, J., \& Smith, J. (1979). Movement and structure as determinants of spatial representations. Journal of Environmental Psychology \& Non-Verbal Behavior, 4, 27-39.

Sherman, R. C., Oliver, C., \& Titus, W. (1980). Verifying environmental relationships. Memory \& Cognition, 8, 555-562.

SIEGEL, A. W., W WITE, S. H. (1975). The development of spatial representations of large scale environments. In H. W. Reese (Ed.), Advances in child development and behavior (Vol. 10; pp. 9-55). New York: Academic Press.

Stevens, A. L., Coupe, P. (1978). Distortions in judged spatial relations. Cognitive Psychology, 10, 422-437.

\section{NOTES}

1. A possible exception to this generalization is an attempt by McNamara et al., (1984, Experiment 2) to control rehearsal separation during the learning of cities on artificial maps by instructing subjects to rehearse and recall town names in a specific sequence. As Clayton and Habibi (1991) have pointed out, however, this procedure may not have completely separated temporal and spatial contiguity, because subjects had to scan the stimulus array to locate the next city in the rehearsal sequence.

2. Substantial differences in error variability between the learning conditions warranted separate analyses (see McNamara et al., 1989, for a similar justification), even though the interactions of distance and region with learning condition were not significant $(F \mathrm{~s}=1.34,1.17,<1$, and $M S_{\mathrm{e}} \mathrm{s}=11,987,16,182$, and 14,107 for distance, region, and distance $X$ region, respectively).

3. This procedure guaranteed that temporal and spatial separation coincided within each region, and in this sense the procedure seemed comparable to that of the free study condition of Experiment 2, in which we observed subjects studying proximal objects one area at a time. For pairs within A or B, the correlation between mean spatial and temporal separation was .96. However, as in everyday environments that are commonly subdivided by features that block direct movement and vision, the same strong association between temporal and spatial separation for some pairs of objects in different regions could not be achieved. The spatial/temporal correlation for A/B pairs was .21. On the average, though, objects in different regions were farther apart both spatially $(M=317 \pm 9 \mathrm{~cm})$ and temporally ( $M=5 \pm .4$ intervening locations) than were objects in the same region $(M \mathrm{~s}=244 \pm 11 \mathrm{~cm}$ and $1.33 \pm .23$ locations).

(Manuscript received June 8, 1990; revision accepted for publication November 26, 1990.) 